\title{
Soutenir le développement de la pensée mathématique des enfants à l'éducation préscolaire : perspectives de recherche
}

\section{HRONIQUE • Recherche étudiante}

\section{Introduction}

Alors qu'un programme-cycle de l'éducation préscolaire (maternelle 4 et 5 ans) est mis en place au Québec (MEQ 2021), les résultats d'une recherche (Marinova et Drainville, 2019) à propos des pratiques enseignantes à cet ordre d'enseignement amènent une importante réflexion chez les enseignantes ${ }^{1}$ concernant les pratiques à privilégier afin de répondre aux besoins des enfants et de soutenir leur développement. En ce sens, des enseignantes reconnaissent avoir besoin de plus de formation et d'accompagnement pour mieux soutenir le développement des enfants (Dumais et Soucy, 2020) et plus spécifiquement en ce qui concerne le développement de la pensée mathématique (St-Jean, 2020). Cette contribution fait état d'une problématique de recherche au regard des pratiques enseignantes, du jeu comme contexte de développement et du développement de la pensée mathématique des enfants. Par la suite, un problème spécifique de recherche est présenté accompagné d'une question de recherche. Puis, l'article se termine en exposant la méthodologie de recherche envisagée.

\section{Les pratiques enseignantes}

Les pratiques enseignantes àl'éducation préscolaire sont principalement issues de deux approches pédagogiques (Marinova et Drainville, 2019). D’une part, l'approche scolarisante amène les enseignantes à privilégier davantage des activités dirigées et un enseignement formel qui visent la préparation des enfants à l'école primaire. Dans cette approche, les enseignantes ont habituellement des pratiques qui visent 
l'acquisition de connaissances par les enfants. D'autre part, l'approche développementale amène plutôt les enseignantes à préconiser des activités instiguées par les enfants comme l'exploration et le jeu. Dans cette approche, les enseignantes ont généralement des pratiques qui visent à étayer le jeu des enfants pour les amener plus loin, et ce, tout en respectant leur unicité.

Comme le jeu symbolique ou de faire semblant constitue l'activité maîtresse par laquelle les enfants d'âge préscolaire se développent et réalisent divers apprentissages (Bodrova et Leong, 2012), celui-ci apparaît comme le contexte de développement par excellence à l'éducation préscolaire. En ce sens, certains résultats de recherche montrent les avantages d'une approche développementale qui met de l'avant le jeu et remettent en question la pertinence d'un enseignement formel à l'éducation préscolaire (Hirsh-Pasek et Golinkoff, 2009; Marinova, Dumais, Moldoveanu et al., 2020). Ainsi, à l'instar de Marinova et Dumais (2019), il s'avère important que les pratiques enseignantes en contexte de jeu «soient encouragées, valorisées et mieux connues du milieu scolaire» (p. 7).

\section{Le jeu comme contexte de développement}

Des chercheurs mentionnent que certaines enseignantes éprouvent de la difficulté à mettre en place des situations de jeu et qu'elles se questionnent quant aux pratiques à privilégier en contexte de jeu (Dumais et Plessis-Bélair, 2017; Marinova, 2009). Cette situation pourrait être associée à un manque de formation relative au jeu ou à une méconnaissance des pratiques enseignantes à privilégier dans celui-ci pour soutenir le développement des enfants (Bouchard et al., 2020; Landry, 2014; Dumais et Soucy, 2020). En ce sens, Laperrière (2016) ainsi que Dumais et Soucy (2020) mentionnent que de nouvelles recherches seraient nécessaires afin de permettre aux enseignantes de mieux comprendre les liens entre le jeu, l'apprentissage et le développement des enfants. De telles recherches pourraient également permettre d'identifier des pratiques enseignantes qui visent à soutenir le jeu et à l'amener à atteindre sa pleine maturité (Laperrière, 2016). D'ailleurs, la fonction symbolique du jeu «atteint son apogée vers l'âge de 7 ou 8 ans » (Marinova, 2016, p. 59), il convient donc d'explorer toutes les avenues qu'il propose pour soutenir le développement des enfants, notamment celui de leur pensée mathématique (Jacob, 2013).

\section{Le développement de la pensée mathématique}

Alors que les habiletés en mathématique apparaissent comme d'importants prédicteurs de la réussite éducative ultérieure des enfants (Duncan et al., 2007), pour bien soutenir le développement de la pensée mathématique de ces derniers, les enseignantes doivent maîtriser les savoirs en lien avec les mathématiques tout comme les pratiques enseignantes à privilégier (Biron, 2016; Deshaies, 2020). Or, certaines études indiquent qu'un manque de connaissances liées aux spécificités des savoirs mathématiques orienterait davantage les enseignantes vers des pratiques scolarisantes (Ginsburg et Ertle, 2008; St-Jean, 2020) au détriment des pratiques associées davantage à une approche développementale. Pourtant, dans cette dernière approche, le jeu symbolique favoriserait le développement des enfants, notamment en ce qui a trait aux habiletés cognitives en lien avec la mathématique (Jacob, 2013; Wyver et Spence, 1999).

2 - Formation et profession 29(3), 2021 


\section{Le problème spécifique de recherche}

Au cours des dernières années, plusieurs chercheurs (ex. : Dumais et Soucy, 2020; Jacob et Charron, 2019; Marinova, Dumais et Leclaire, 2020) ont réalisé des études dans lesquelles ils ont accompagné des équipes d'enseignantes dans la mise en place de moments de jeu où les enseignantes soutenaient le développement des enfants. La majorité de ces recherches ont porté sur le développement du langage. À notre connaissance, aucune recherche québécoise n'a été menée en ce qui concerne les pratiques enseignantes à l'éducation préscolaire en contexte de jeu pour soutenir le développement de la pensée mathématique des enfants. Ainsi, mener une telle recherche permettrait d'apporter de nouvelles connaissances sur le plan scientifique.

\section{La question de recherche}

Parmi les questions qui demeurent sans réponse et qui demandent d'être étudiées, la question de recherche suivante a été retenue : "Quelles pratiques enseignantes permettent de soutenir le développement de la pensée mathématique des enfants en contexte de jeu à l'éducation préscolaire?»

\section{La méthodologie envisagée}

Afin de répondre à cette question, une recherche-action qui s'appuie sur les préoccupations d'enseignantes de l'éducation préscolaire qui souhaitent changer certaines de leurs pratiques est envisagée. Cette recherche permettrait de documenter les pratiques enseignantes et de faire ainsi état d'éventuels changements de pratiques de la part des participantes.

\section{Conclusion}

Les résultats de cette recherche apporteraient sans doute un éclairage nouveau concernant certaines pratiques enseignantes en contexte de jeu qui permettent de soutenir le développement de la pensée mathématique des enfants.

\section{Note}

1 Le terme «enseignante» est privilégié plutôt que le terme «enseignant» dans cet article, et ce, pour souligner la plus grande représentativité des femmes dans les milieux éducatifs, notamment à l’éducation préscolaire (MEES, 2019).

\section{Bibliographie}

Biron, D. (2016). Développer la pensée mathématique des enfants à l'éducation préscolaire. Dans C. Raby et A. Charron (dir.), Intervenir à l'éducation préscolaire : pour favoriser le développement global de l'enfant. (2éd., p. 10-21). Les éditions CEC.

Bodrova, E. et Leong, D. J. (2012). Les outils de la pensée. L'approche vygostkienne dans l'éducation à la petite enfance. Presses de l'Université du Québec. 
Bouchard, C., Bergeron-Morin, L., Parent, A.-S., Charron, A. et C. Julien (2020). Soutien du langage oral et de l'émergence de l'écrit des enfants en situation de jeu de faire semblant : un contexte propice, mais peu exploité en maternelle 5 ans. Approche Neuropsychologique des Apprentissage chez l'Enfant (A.N.A.É.), 165, 1-11.

Deshaies, I. (2020). L'apprentissage des mathématiques au préscolaire 4 ans et 5 ans. Dans I. Deshaies et J.-M. Miron (dir.), Tisserands d'enfance : le développement de l'enfant de 4 et 5 ans (p. 105-141). JFD Éditions.

Dumais, C. et Plessis-Bélair, G. (2017). Le jeu symbolique : contexte de développement du langage oral d'enfants de la maternelle 4 ans et 5 ans en milieu plurilingue et pluriethnique. Dans C. Dumais, R. Bergeron, M. Pellerin et C. Lavoie (dir.), L'oral et son enseignement : pluralité des contextes linguistiques (p. 175-200). Éditions Peisaj.

Dumais, C. et Soucy, E. (2020). Des besoins de formation continue d'enseignantes de la maternelle 4 ans et 5 ans au Québec : constats issus de recherches. Revue internationale de communication et de socialisation, 7(1-2), 106-127. https:// www.revuerics.com/wp-content/uploads/2021/07/RICS 2020-Vol-7-1 Dumais-et-Soucy-La-maternelle-4-ansa\%CC\%80-temps-plein-14-11-2020.pdf

Duncan, G. J., Dowsett, C. J., Claessens, A., Magnuson, K., Huston, A.C., Klebanov, P. et Japel, C. (2007). School readiness and later achievement. Developmental Psychology, 43(6), 1428-1446. https://doi.org/10.1037/0012-1649.43.6.1428

Ginsburg, H. et Ertle, B. (2008). Knowing the mathematics in early childhood mathematics. Dans O. Saracho et B. Spodek (dir.), Contemporary perspectives on mathematics in early childhood education (p. 45-66). Information Age Publishing.

Hirsh-Pasek, K. et Golinkoff, R. M. (2009). Pourquoi Jouer = Apprendre. Dans Encyclopédie sur le développement des jeunes enfants. https://www.enfant-encyclopedie.com/jeu/selon-experts/pourquoi-jouer-apprendre

Jacob, É. (2013). L'implication des mères atikamekw dans le jeu symbolique de leur enfant. Dans G. Maheux et R. Gauthier (dir.), La formation des enseignants inuit et des Premières nations : problématiques et pistes d'action (p. 9-29). Presses de l'Université du Québec.

Jacob, É. et Charron, A. (2019). Enjeux d'une recherche collaborative menée avec trois enseignantes atikamekws à l'éducation préscolaire. Revue hybride de l'éducation, 2(2), 1-23. https://doi.org/10.1522/rhe.v2i2.191

Landry, S. (2014). Favoriser le développement de la pensée sociale par l'étayage du jeu symbolique chez des enfants qui fréquentent la maternelle cinq ans. [thèse de doctorat, Université Laval]. CorpusUL. http://hdl.handle.net/20.500.11794/24789

Laperrière, A. (2016). La place du jeu à la maternelle. Réseau d'information pour la réussite éducative. http://rire.ctreq.qc.ca/ wp-content/uploads/2016/01/Article-RIRE-Publisher-5 LB.pdf

Marinova, K. (2009). L'acquisition du langage dans le jeu de rôles au préscolaire. Québec français, 155, 62-63.

Marinova, K. (2016). Le développement de la pensée mathématique dans le jeu de rôles. Dans K. Marinova et D. Biron (dir.), Mathématiques ludiques pour les enfants de 4 à 8 ans (p. 57 à 88). Presses de l'Université du Québec.

Marinova, K. et Drainville, R. (2019). La pression ressentie par les enseignantes à adopter des pratiques scolarisantes pour les apprentissages du langage écrit à l'éducation préscolaire. Revue canadienne de l'éducation, 42(3), 605-634. https:// journals.sfu.ca/cje/index.php/cje-rce/article/view/3490

Marinova, K. et Dumais, C. (2019). Lire et écrire dans les situations d'apprentissage issues du jeu symbolique : les résultats d'une recherche. Revue préscolaire, 57(4), 5-7.

Marinova, K., Dumais, C. et Leclaire, É. (2020). Le rôle des enseignantes lors du jeu symbolique : réflexions au sein d'une communauté de pratique d'enseignantes de l'éducation préscolaire. Revue Internationale de Communication et Socialisation (RICS), 7(1-2), 128-154. https://www.revuerics.com/wp-content/uploads/2021/07/RICS 2020-Vol-71 Marinova-et-al-Le-ro\%CC\%82le-des-enseignantes-lors-du-jeu-symbolique-14-11-2020.pdf

Marinova, K., Dumais, C., Moldoveanu, M., Dubé, F. et Drainville, R. (2020). Les pratiques enseignantes pour soutenir les premiers apprentissages de la langue écrite à l'éducation préscolaire : entre l'approche développementale et l'approche scolarisante. Revue des sciences de l'éducation de McGill, 55(2), 352-375. https://doi.org/10.7202/1077972ar

Ministère de l'Éducation du Québec (2021). Programme-cycle de l'éducation préscolaire. Gouvernement du Québec. http:// www.education.gouv.qc.ca/fileadmin/site web/documents/education/jeunes/pfeq/Programme-cycle-prescolaire.pdf 
Ministère de l'Éducation et de l'Enseignement supérieur (2019). Rapport annuel 2018-2019. Gouvernement du Québec. https://cdn-contenu.quebec.ca/cdn-contenu/adm/min/education/publications-adm/rapport-annuel-de-gestion/ MEES RAG 2018-2019.PDF?1570037423

St-Jean, C. (2020). La qualité des interactions enseignante-enfants et le développement du raisonnement spatial à la maternelle quatre ans temps plein en milieu défavorisé. [thèse de doctorat, Université du Québec en Outaouais]. Archipel. http:// archipel.uqam.ca/id/eprint/14301

Wyver, S.R. et Spence, S.H. (1999). Play and divergent problem solving: Evidence supporting a reciprocal relationship. Early Education and Development, 10(4), 419-444.

\section{Pour citer cet aricle}

Nolin, R. (2021). Soutenir le développement de la pensée mathématique des enfants à léducation préscolaire : perspective de recherche [Chronique]. Formation et profession, 29(3), 1-5. http://dx.doi.org/10.18162/fp.2021.a236 